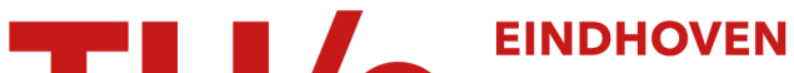 UNIVERSITY OF TECHNOLOGY
}

\section{Organic pressure sensing surfaces fabricated by lamination of flexible substrates}

\section{Citation for published version (APA):}

Fattori, M., Abdinia, S., Cantatore, E., Pauer, G., Stadlober, B., Gold, H., Socratous, J., \& Torricelli, F. (2018). Organic pressure sensing surfaces fabricated by lamination of flexible substrates. IEEE Transactions on Components, Packaging and Manufacturing Technology, 8(7), 1159-1166. [8307231].

https://doi.org/10.1109/TCPMT.2018.2799700

DOI:

10.1109/TCPMT.2018.2799700

Document status and date:

Published: 01/07/2018

\section{Document Version:}

Accepted manuscript including changes made at the peer-review stage

\section{Please check the document version of this publication:}

- A submitted manuscript is the version of the article upon submission and before peer-review. There can be important differences between the submitted version and the official published version of record. People interested in the research are advised to contact the author for the final version of the publication, or visit the $\mathrm{DOI}$ to the publisher's website.

- The final author version and the galley proof are versions of the publication after peer review.

- The final published version features the final layout of the paper including the volume, issue and page numbers.

Link to publication

\section{General rights}

Copyright and moral rights for the publications made accessible in the public portal are retained by the authors and/or other copyright owners and it is a condition of accessing publications that users recognise and abide by the legal requirements associated with these rights.

- Users may download and print one copy of any publication from the public portal for the purpose of private study or research.

- You may not further distribute the material or use it for any profit-making activity or commercial gain

- You may freely distribute the URL identifying the publication in the public portal.

If the publication is distributed under the terms of Article $25 \mathrm{fa}$ of the Dutch Copyright Act, indicated by the "Taverne" license above, please follow below link for the End User Agreement:

www.tue.nl/taverne

\section{Take down policy}

If you believe that this document breaches copyright please contact us at:

openaccess@tue.nl

providing details and we will investigate your claim. 


\title{
Organic Pressure Sensing Surfaces Fabricated by Lamination of Flexible Substrates
}

\author{
Marco Fattori, Sahel Abdinia, Gernot Pauer, Barbara Stadlober, Herbert Gold, Josephine Socratous, \\ Fabrizio Torricelli, and Eugenio Cantatore, Fellow, IEEE
}

\begin{abstract}
This paper presents the design and experimental characterization of large-area active matrixes on foil for pressure sensing applications. Frontend circuits based on organic thin-film transistors on a flexible substrate are laminated with a foil hosting screen-printed PDVF-TrFE piezo sensors to create the complete flexible sensing systems with $6 \times 10$ sensing elements on a $16.5 \mathrm{~cm} \times 27.5 \mathrm{~cm}$ area. After defining the specifications based on the application scenarios, and designing two different frontend matrixes ( $A$ and $B$ ), the performance of the sensing surface $B$ has been investigated in simulation. Numerical results show a readout speed of $5 \mathrm{kframe} / \mathrm{s}$ and $78.6 \mathrm{~dB}$ maximum $\mathrm{SNR}$ with impact forces up to $50 \mathrm{kN}$. Experiments made with a prototype based on frontend A confirm that the system can correctly reconstruct the impact profile of forces up to $50 \mathrm{kN}$ obtained in a drop tower setup. Measurements of the frontend circuit $B$ show an input equivalent noise of $451 \mu \mathrm{V}_{\text {rms }}$ and a $5 \%$ settling time of $13.8 \mu \mathrm{s}$, both adequate for the specified applications.
\end{abstract}

Index Terms- Flexible electronics; Pressure sensing; Piezoelectric sensors; Large-Area electronics; OTFTs.

\section{INTRODUCTION}

$\mathrm{A}^{\mathrm{n}}$ major concern in the public healthcare domain is the management of domestic incidents involving elderly people. Events like falls often cause serious physical injuries and long hospitalizing time, involving high societal and economic costs. The use of automatic monitoring and fall detection systems in domestic environments could enable prompt assistance, reducing the negative effects of the fall. Indeed, people who fell are often unable to recover autonomously the standing position and ask for help.

In the recent years, the fall detection problem has been widely analyzed in the literature leading to the development of a large variety of solutions. Those are typically organized in two major categories: wearable and non-wearable solutions. Most of the systems belonging to the first class exploit the use of Inertial Measurement Units (IMUs) for fall detection [1]. Wearable devices are typically placed on wrists, ankle or waist and therefore the requirement of comfort is of primary concern. The positioning of the sensors on the body has a significant impact on the system detection capability and on the level of acceptance by the user. Non-wearable systems monitor the

M. Fattori, S. Abdinia and E. Cantatore are with the Eindhoven University of Technology, Eindhoven, The Netherlands (e-mails: M.Fattori@tue.nl, S.Abdinia@tue.nl, E.Cantatore@tue.nl). G. Pauer is with Altran Concept Tech, Gratkorn, Austria (e-mail: Gernot.Pauer@altran.com). B. Stadlober and H. Gold, are with Johanneum Research, Graz, Austria (e-mails: domestic environment by means of e.g. radar [2], vision [3] and acoustic [4] sensors. Several of these systems suffer from privacy issues. An approach that is more respectful of privacy is the use of smart floors, which exploit pressure-sensing surfaces [5] to detect falls. Ambient monitoring solutions require typically high computational resources, and high costs, restricting their applicability to small indoor environments only.

Organic materials offer an attractive solution to overcome these limitations, enabling the manufacturing of pressure sensor systems over large-area at very low cost. Indeed, the low process temperatures typical of organic Thin Film Transistor (OTFT) technology enable the production of electronic functions on inexpensive flexible plastic substrates. The compatibility with high throughput additive printing processes leads to a further cost reduction. Moreover, plastic substrates are especially suited for pressure sensing applications, as they are rugged and can tolerate bending and large compressive forces.

Active matrixes based on organic transistors have been already proved to detect spatial pressure distribution in several applications, such as electronic artificial skin [6-8], and tactile sensors [9-11]. In these applications, low sensitivity and fast response have been the main concerns. However, the possibility of achieving high dynamic ranges in pressure measurements has not been proven yet. The demonstration of low cost solutions for applications where large dynamic range and fast response are required could bring organic electronics to the market of home monitoring, healthcare, and human-machine interfaces for smart buildings, to mention just a few examples.

In this work, we design pressure-sensing surfaces suitable for these applications, which exploit the combination of piezoelectric sensors [10] with optimal mechanical properties even for large applied forces, and active matrixes manufactured with Organic Thin Film Transistors (OTFTs) [12].

The paper is further organized as follows: the system specifications are derived in section II; the design of the active matrixes is described in section III; the characterization of the pressure sensor is reported in section IV; simulations and numerical results of the pressure sensing surfaces are discussed

Barbara.Stadlober@joanneum.at, Herbert.Gold@joanneum.at). J. Socratous is with FlexEnable, Cambridge, United Kingdom (e-mail: Josephine.Socratous@flexenable.com). F. Torricelli is with University of Brescia, Brescia, Italy (e-mail: Fabrizio.Torricelli@unibs.it). 
in section V; measurements results are presented in section VI, and conclusions are drawn in section VI.

\section{SySTEM SPECIFICATIONS}

The sensors employed in this work are based on the ferroelectric polymer $\mathrm{P}(\mathrm{VDF}-\mathrm{TrFE})$, which exhibits piezoelectric properties. A screen-printing process is used to fabricate the sensors on a flexible $175-\mu$ m-thick PET substrate. The ferroelectric polymer is sandwiched between the top and bottom electrodes made of either conductive polymer PEDOT:PSS or carbon. A reproducible permanent polarization is then obtained by hysteresis poling [10]. More details on the sensor fabrication are provided in [10]. The main characteristics of the sensors used in this work are reported in Table I.

TABLE I

SPECIFICATIONS OF PIEZOELECTRIC SENSORS

\begin{tabular}{cccc}
\hline \hline P(VDF-TrFE) sensor & Symbol & Value & Unit \\
\hline Piezoelectric coefficient & $\mathrm{d}_{33}$ & 32 & $\mathrm{pC} \mathrm{N}^{-1}$ \\
Capacitance per unit area & $\mathrm{C}_{0}$ & 1.2 & $\mathrm{nFcm}^{-2}$ \\
\hline \hline
\end{tabular}

The force applied generates a charge at the surface of the sensor ferroelectric material, thanks to its piezoelectric response. At first approximation the total charge delivered results proportional to the force applied along the direction perpendicular to the sensor surface, through the piezoelectric coefficient $\mathrm{d}_{33}$ [13].

The characteristics of printability over large-areas, low cost and good uniformity [10], together with the intrinsic piezoelectric sensitivity of the polymer, make this sensor perfectly suited for the targeted applications. The sensor matrix is integrated on a foil called frontplane. The frontend and addressing electronics are based on a commercial OTFT technology [14]. The frontend matrix (called here backplane) shares the same pitch as the sensor matrix (Fig. 1). The readout is performed addressing a row of sensors at the time. The rowselect signal will be provided by integrated OTFT line drivers, while the columns are connected to silicon readout electronics on a PCB board (Fig. 1). In the rest of this section, more detailed specifications for the pressure-sensing surfaces will be derived.

\section{System Integration}

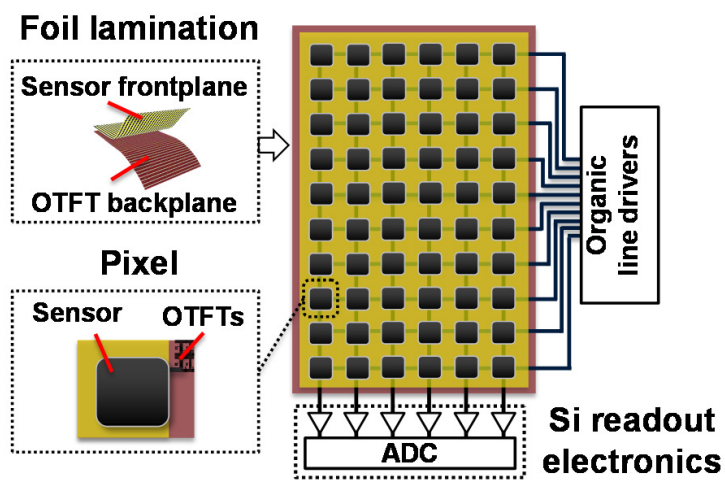

Fig. 1 System integration of the proposed pressure sensing surfaces.

In this work, two frontend matrixes for pressure sensing featuring $6 \times 10$ elements on a $16.5 \mathrm{~cm} \times 27.5 \mathrm{~cm}$ area are presented. The first one (called matrix A) is used to demonstrate the feasibility of the proposed system integration for the target applications. The second one (matrix B), features a more complex backplane design in order to improve the performance and reliability of the system. The frontplane is fully compatible with both matrixes. Indeed, the cell size of each matrix is 27.5 $\mathrm{mm} \times 27.5 \mathrm{~mm}$ and the active area of the sensors is chosen to be $18 \mathrm{~mm} \times 18 \mathrm{~mm}$. This is dictated in first place by the need to access each sensor element separately during the poling process. Therefore, part of the cell area must be reserved to draw the poling lines. In second place, direct mechanical stress on the organic transistors in the frontend circuit should be avoided in order to preserve the correct functionality of the pixels. Thus, some clearance from the impact area must be adopted when placing the OTFTs. The layout of a pressuresensitive pixel is shown in Fig. 1, and in greater detail, in Fig. 2. It is worth noticing that the charge generated by the sensor is, at first approximation, independent from the sensor size when the impact area and the sensor active area coincide. Due to the mechanical arrangement of our sensing surface, as it will be detailed later in Section VI, the force F is uniformly distributed on the sensor area $A_{s}$, and the corresponding pressure $\mathrm{p}$ can be calculated as

$$
\mathrm{p}=\frac{\mathrm{F}}{\mathrm{A}_{\mathrm{s}}}
$$

In order to develop prototypes that are suitable for several applications in the market of the force detection and pressure mapping solutions, a dynamic range of $60 \mathrm{~dB}$ has been chosen, with a maximum applicable force of $50 \mathrm{kN}$. In order to further increase the versatility of our solution, not only a large dynamic range is specified, but also a fast response in the impact detection. For this reason, a readout speed of $5 \mathrm{kframes} / \mathrm{s}$ will be required for the reconstruction of the impact profiles.

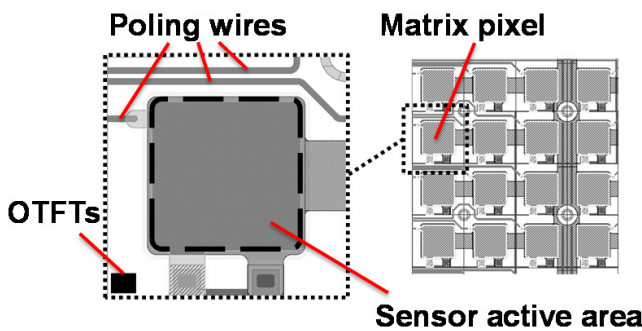

Fig. 2 Pixel matrix layout for the pressure sensing surfaces.

The system specifications for the active matrix are summarized in Table II.

TABLE II

SYSTEM SPECIFICATIONS

\begin{tabular}{ccc}
\hline \hline Pressure sensing surfaces & Value & Unit \\
\hline Matrix elements & $6 \times 10$ & - \\
Cell size & 7.56 & $\mathrm{~cm}^{2}$ \\
Sensor active area & 3.24 & $\mathrm{~cm}^{2}$ \\
Sensor capacitance & 3.88 & $\mathrm{nF}$ \\
Maximum applicable force & 50 & $\mathrm{kN}$ \\
Dynamic Range & 60 & $\mathrm{~dB}$ \\
Frame rate & 5 & $\mathrm{kframe} / \mathrm{s}$ \\
Row addressing time & 20 & $\mu \mathrm{s}$ \\
\hline \hline
\end{tabular}




\section{DESIGN OF ThE MATRIX BACKPLANES}

According to the specifications, relatively high forces might be applied to the sensing surface. In this case, due to the high piezoelectric coefficient, a significant amount of charge will be delivered by the sensors. The piezoelectric sensor can be electrically modelled as a current source, having a finite internal resistance $R_{S}(\sim G \Omega)$, that accounts for the sensor leakage (Fig. 3 ). If no low-impedance path is provided, the charge will accumulate on the sensor capacitor $\mathrm{C}_{\mathrm{S}}$ (Fig. 3). It is evident that complete integration of the sensor charge on the sensor capacitor is not feasible. Indeed, in the worst-case scenario, considered the specs in Table I and II, the generated peak voltage across the sensor would be approximately given by

$$
\mathrm{V}_{\text {peak }}=\frac{\mathrm{F}_{\text {peak }} \mathrm{d}_{33}}{\mathrm{C}_{\mathrm{S}}} \approx 420 \mathrm{~V}
$$

where $F_{\text {peak }}$ is the maximum value of the applied force. Such a high voltage would permanently damage the OTFTs in the frontend.

This issue could be alleviated by increasing the sensor capacitance. As discussed in section II, the sensor dimensions cannot be increased. Hence, the only way to achieve larger capacitance is by means of additional capacitors implemented in the backplane. However, circuit structures distributed over a large area are prone to the formation of hard defects, shorts for example, and therefore are detrimental for the yield. The solution proposed in this work is based on the use of a small resistor $\mathrm{R}$ in parallel to the sensor, fabricated on the metal layer of the backplane, to provide a low-impedance path for the sensor charge, as shown in Fig. 3. If the resistance value is correctly chosen, no passive integration of the current occurs on the sensor capacitance within the bandwidth of interest. Therefore, the voltage across the sensor results limited within a safe range. Its maximum value will be approximately given by

$$
\mathrm{V}_{\text {peak, max }}=\left.\mathrm{R} \frac{\partial\left(\mathrm{d}_{33} \mathrm{~F}\right)}{\partial \mathrm{t}}\right|_{\max }
$$

Where $\mathrm{F}$ is the force applied to the sensor. The cut-off frequency of the first order low-pass filter and the voltage output swing are determined by R: a good trade-off is obtained by choosing a resistance of $5 \mathrm{k} \Omega$. Indeed, with this choice, the cut-off frequency is set to $\sim 8 \mathrm{kHz}$ (which is sufficiently fast to enable the specified frame rate), while the maximum output swing will be approximately $\pm 5 \mathrm{~V}$. In addition, when an impact with the maximum allowed force is considered, the peak value of the electric power on the resistor is $5 \mathrm{~mW}$. In order to fabricate the resistor on the backplane metal layer, 167 squares are required, which corresponds to an area of $800 \mu \mathrm{m} \times 4700 \mu \mathrm{m}$. The estimation of the maximum output swing together with the dynamic range specification are used to define the in-band input referred noise requirement for the frontend electronics. For this scope, we will approximate, the sensor output, by means of its fundamental harmonic component only. Under these conditions, the Signal-to-Noise-Ratio (SNR), at the input of the frontend electronics can be expressed as:

$$
\mathrm{SNR}=\frac{\mathrm{V}_{\text {peak }}^{2}}{2 \cdot \mathrm{V}_{\mathrm{n}, \mathrm{rms}}^{2}}
$$

where $\mathrm{V}_{\text {peak }}$ is the amplitude of the fundamental harmonic and $\mathrm{V}_{\mathrm{n}, \mathrm{rms}}$ is the rms value of the integrated input equivalent noise. Given the shape of the expected applied force, the value chosen for $\mathrm{R}$, the coefficient $\mathrm{d}_{33}$ and the DR specified in Table II, if a minimum SNR of $10 \mathrm{~dB}$ is assumed to be sufficient to reconstruct the impact forces, the equivalent noise level that can be tolerated at the input of the frontend is at maximum $1.1 \mathrm{mV}_{\text {rms. }}$ This, according to [15], leads to an estimated Limit Of Detection (LOD) of $3.3 \mathrm{mV}$ which corresponds approximately to an impact force of $33 \mathrm{~N}$.

Since the amplitude of the signal is rather high, noamplification is required in the frontend. Two different pressure sensing backplanes (matrix A and matrix B) have been designed in this work, as stated in the section II.

Matrix A aims to demonstrate the system integration and validate the proposed readout approach. The design of the pixel frontend is thus reduced to the active matrix addressing only, as shown in Fig. 3(a). A matrix readout in voltage mode is preferred, rather than a current-mode one, due to the lower pixel complexity that it makes possible. It should be noticed here that the TFT counts in the pixel must be kept as low as possible, to ensure good yield on large sensing surfaces.

The loading effect of the readout electronics $(10 \mathrm{pF})$ together with the parasitic due to the column interconnections $(10 \mathrm{pF})$ and the active addressing transistors $(15 \mathrm{pF})$, are accounted for by the loading capacitance $\left(\mathrm{C}_{\mathrm{CLM}}\right)$ (Fig. 3). Its value is analytically estimated to be approximately $35 \mathrm{pF}$, based on the geometries of devices and interconnections. In order to enable a fast active matrix readout that satisfies our specifications, the time constant introduced by the on-resistance of $\mathbf{M}_{1}$ and the capacitor $\mathrm{C}_{\mathrm{CLM}}$ must be a few times shorter than the row addressing time $(20 \mu \mathrm{s})$. The size of the transistor $\mathrm{M}_{1}$ must be chosen accordingly.

(a)

Sensor model Frontend

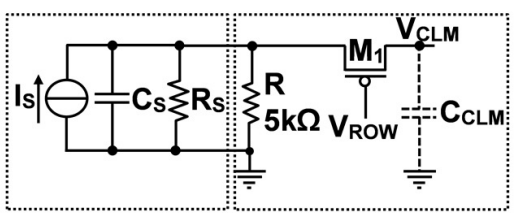

(b)

\section{Frontend}

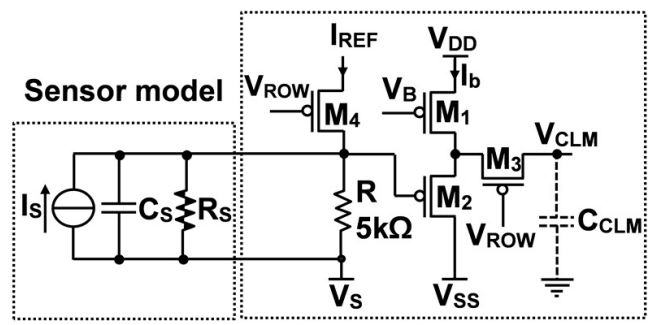

Fig. 3 Sensor electrical model and schematic of the proposed pixel frontends, for the $6 \times 10$ matrix A (a) and matrix B (b). 
The pixel schematic described until now, although simple and compact, is affected by an important crosstalk issue. Let us consider the worst-case scenario, in which two consecutive pixels in a column are hit by the maximum $\left(\mathrm{F}_{\max }\right)$, and minimum specified force $\left(\mathrm{F}_{\min }\right)$. If we assume for the sake of simplicity that the shape of the force pulse is the same, using equation (3) we can write:

$$
\mathrm{V}_{\text {peak, max }}=\frac{\mathrm{F}_{\text {max }}}{\mathrm{F}_{\text {min }}} \cdot \mathrm{V}_{\text {peak, min }}
$$

In this scenario, the parasitic capacitance $\mathrm{C}_{\mathrm{CLM}}$ is charged to $\mathrm{V}_{\text {peak,max }}$ and then discharged to a voltage $\mathrm{V}_{\text {meas }}$ that will be measured on the pixel. The normalised error between the correct value of $\mathrm{V}_{\text {peak,min }}$ and its measured value affected by cross-talk, $\mathrm{V}_{\text {meas }}$ is:

$$
\varepsilon=\frac{\mathrm{V}_{\text {meas }}-\mathrm{V}_{\text {peak, min }}}{\mathrm{V}_{\text {peak, min }}}
$$

For $\mathrm{C}_{\mathrm{CLM}}=35 \mathrm{pF}$ and using further the specifications in Table II, $\varepsilon$ can be found to be equal to $750 \%$, which means that the measurement is completely corrupted by the cross-talk.

To avoid this significant cross-talk issue, the second pressure sensing frontend (matrix B), integrates in each pixel a voltage buffer implemented with a source follower $\left(\mathrm{M}_{1}-\mathrm{M}_{2}\right.$ in Fig. 3(b)), which is used to decouple the sensor from the column capacitance. The pass transistor $\mathrm{M}_{3}$ is added to enable active addressing of each pixel. The drawback of this solution is an increased OTFT count in the pixel. Besides, assuming $\mathrm{C}_{\mathrm{CLM}}=$ $35 \mathrm{pF}$ and based on OTFT simulations, a bias current $\mathrm{I}_{\mathrm{b}}$ of $90 \mu \mathrm{A}$ is required in the follower to fulfil the specification on time response.

Large parameter variability is an issue that severely affects large-area electronics. Therefore a calibration procedure is required to eliminate offset and gain errors when using the pixel with source follower. To achieve this, an additional pass transistor $\mathrm{M}_{4}$ is introduced in the frontend circuit (Fig. 3(b)). This enables the injection of a reference current $I_{\text {REF }}$ in the resistor R. From the output voltage measured for two different values of $\mathrm{I}_{\mathrm{REF}}$, the offset and gain errors can be estimated for each pixel.

The frontend pixel layouts for the matrix A and B are shown in Fig. 4 (a), and (b), respectively.

(a)

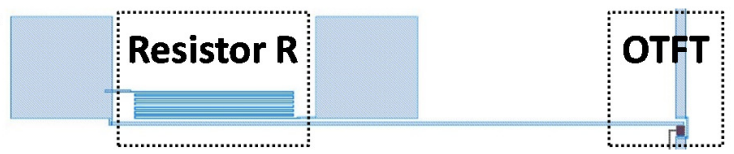

(b)

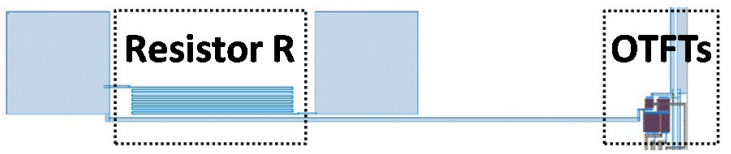

Fig. 4 Pixel frontend layouts for the matrix A (a), and the matrix B (b).
In the voltage readout approach, the signal sensed by the frontend is proportional to the derivative of the applied force with respect to time. Thus, after digitalization, a numerical integration must be performed to determine the corresponding applied impact-force/pressure.

\section{SENSOR CHARACTERIZATION}

The large dynamic range required for the pressure/impactforce sensing surface poses severe requirements in terms of mechanical robustness and durability of the materials. Repetitive impact tests have been performed on the sensors, by means of a drop tower, in order to assess the reproducibility of the response and the lifetime. No apparent degradation has been observed after several impacts, with reference forces in the range of 30-50 kN. Three calibrated force gauges, have been placed underneath a circular aluminum disk, at the base of the drop tower. The correct positioning of the reference sensors are chosen according to the nodal points of the lowest frequency eigen-oscillation modes of the round plate. In this way, unwanted signal contributions due to the oscillation of the plate itself, are reduced. During the impacts, the sensor and the calibrated force gauges, are simultaneously measured. The sensor calibration curve is extracted as linear least squares fitting of the crossplot between the sensor output and the reference force signal Fig. 5. Hence, the slope of the linear fitting in Fig. 5 defines the scaling factor to determine the calibration of the sensor and expresses its response in terms of input force (Fig. 6). The normalized rms error between the calibrated sensor response and the measured reference force (Fig. 6) is $11.5 \%$.

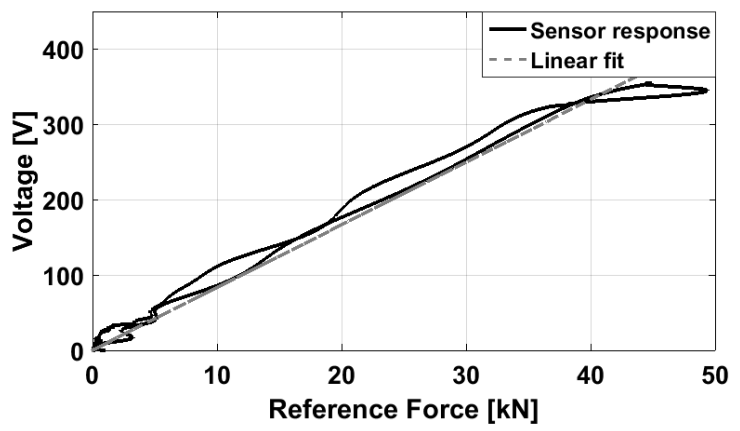

Fig. 5 Crossplot between measured sensor response and reference force (continuous line) and extracted sensor calibration curve (dashed grey).

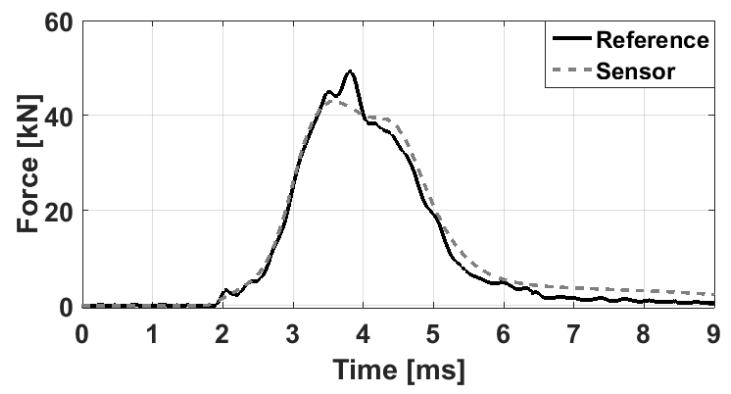

Fig. 6 Calibrated piezoelectric sensor response (dotted grey line) to an impact test with a reference peak force of $50 \mathrm{kN}$ (black). 


\section{BEHAVIORAL SimULATIONS}

Simulations of the pixel response for the matrix B have been performed using a commercial CAD framework. The noise performance of the frontend has been evaluated with circuit simulations, assuming the source follower $\mathrm{M}_{1}-\mathrm{M}_{2}$ (Fig. 3(b)) at the nominal bias conditions, and $\mathrm{M}_{3}$ permanently turned on. In these conditions an equivalent input noise of $413 \mu \mathrm{V}_{\mathrm{rms}}$ is obtained, which according to (3), leads to an SNR of 78.6dB. This value, is more than sufficient to ensure a $60 \mathrm{~dB}$ dynamic range in the detection of the input forces.

Furthermore, in order to validate the matrix functionality at nominal readout speed, a dynamic circuit simulation has been also performed. Several current profiles recorded during the calibration tests have been used in the sensor equivalent circuit of each pixel to simulate the impact tests. Next, the matrix columns have been readout at a speed of $5 \mathrm{kframe} / \mathrm{s}$, and the output streams demultiplexed. The signals coming from the different pixels have been upsampled by a factor ten, and reconstructed through interpolation filtering. The result obtained for one pixel is reported in Fig. 7 together with the original current profile. From the simulation results, a good accuracy in the signal reconstruction is observed. Indeed, the normalized rms error between reconstructed and actual input is lower than $5 \%$. At last, the time evolution of the applied force on the sensor is obtained by means of a numerical integration. The result is shown in Fig. 8.

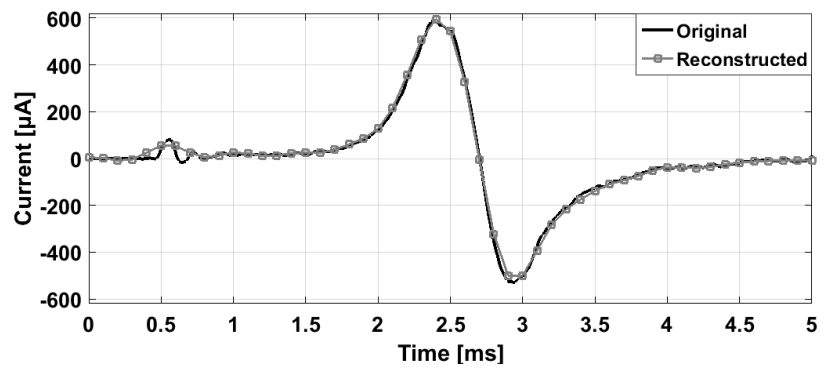

Fig. 7 Simulated response of a front pixel matrix. Comparison between the original current profile (black) and the reconstructed one (gray squares).

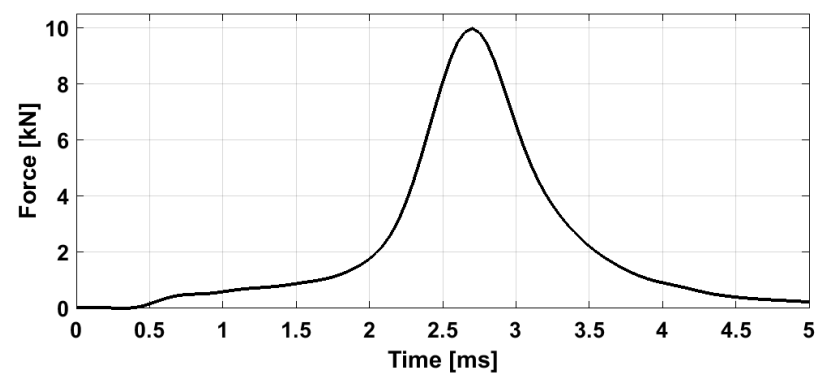

Fig. 8 Applied force profile, reconstructed by numerical integration.

\footnotetext{
${ }^{1}$ It should be noted here that Matrix A does not provide calibration support in the backplane to measure the resistor R. Thus, the value of $\mathrm{R}$ can only be determined via a calibration of the complete assembly sensor + pixel frontend.
}

\section{MeAsurements Results}

\section{A. Measurement setup}

The matrix A backplane described in section III, has been manufactured and integrated with the rest of the system parts. The frontplane and the backplane have been laminated using patches of conductive adhesive to ensure the electrical connectivity between the foils. Next, a PCB with off-the-shelf components has been designed to interface the matrix with the data acquisition system. An array of six voltage buffer amplifiers is used to readout the matrix columns in voltage mode (high impedance) and drive the loading capacitances introduced by the coaxial cables, connecting to the next stage of the chain. In addition, the PCB provides the line addressing signals to enable periodically the matrix rows to be readout. Finally, the six channels are simultaneously digitalized with the Dewesoft Dewe-43. The digital post-processing techniques described in the section $\mathrm{V}$ are then applied to reconstruct the curve of the applied force on each pixel.

From the mechanical point of view, a few aspects have been considered for the arrangement of the measurements setup. An aluminum adaptor has been fabricated to transfer the applied force only on the active area of the sensors protecting the frontend electronics from damages during the impact tests. Hence, the system on foil is sandwiched between one side of the adaptor, containing milled structure with protruding areas, and several single transfer plates on the other side (Fig. 9 (c)). In particular the transfer plates can be arbitrarily mounted on the adaptor, to allow the distribution of the forces on single or multiple pixels. Indeed, when the pixel calibration is performed, only one transfer plate at the time is required, applying force to just one pixel. In the nominal working conditions instead, all the transfer plates are mounted on the adaptor since in principle, all the matrix elements could be mechanically excited at the same time.

The need for calibration is twofold: first, it is required to compensate the variability of the sensor parameters, which are intrinsically more pronounced in printing manufacturing processes. Second, it allows to determine the value of the resistor $\mathrm{R}$ introduced in the frontend, thus correcting the pixel frontend gain error ${ }^{1}$.

At last, drop tower tests have been used to evaluate the prototype performance. For each testing condition, several impacts are recorded to assess the reproducibility of the results.

\section{B. Matrix A Prototype Performance Evaluation}

In this subsection the experimental results recorded during impact tests on a sub set of $2 \times 2$ pixels are presented. The pixels under test belong to the matrix columns $\mathrm{C} 2, \mathrm{C} 3$, and rows R2, $\mathrm{R} 3$. The first step of the evaluation process is the extraction of the sensor calibration curves for the active pixels. One transfer plate at a time is mounted in the adaptor, so that the force can only be transferred to the sensor under test. The impactor sled (mass of $\sim 1.5 \mathrm{Kg}$ ) having a flat impact surface, is dropped from 
a height of $100 \mathrm{~cm}$ in order to obtain reference force profiles with symmetrical shape and maximum amplitude of about $20 \mathrm{kN}$. A rubber cube having $2 \mathrm{~cm}$ edge, is than placed on top of the aluminum adaptor to increase the damping effect and broaden the shape of the applied force profile.
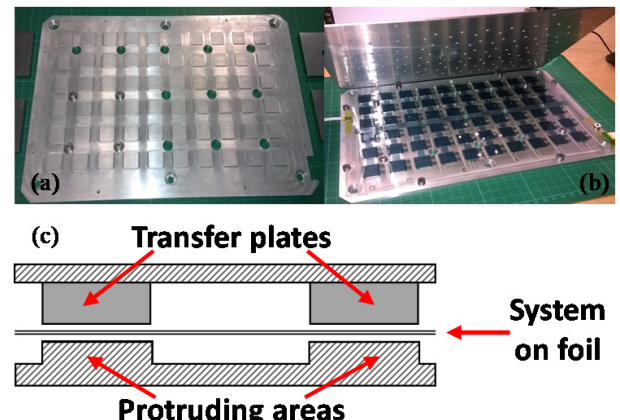

Fig. 9 Aluminum adapter and pressure sensing surface prototype. (a) Milled structure with protruding areas for force distribution. (b) Pressure sensing surface arrangement inside the adaptor. (c) Cross section of the aluminum adaptor.

The data recorded from the prototype during the tests is digitally processed to extract the calibration curve from the force detected on each pixel.

Next, four individual transfer plates are introduced in the aluminum adaptor to engage the entire sub set of four pixels. An asymmetric distribution of the applied force over the sensing surface is obtained by placing a rubber cube above one of the active sensors. In this scenario, the applied force can be calculated as superposition of the single contributions measured by the four sensors, since no additional load paths are active. The comparison between the reference and resultant force is shown in Fig. 10 together with the single force contributions.

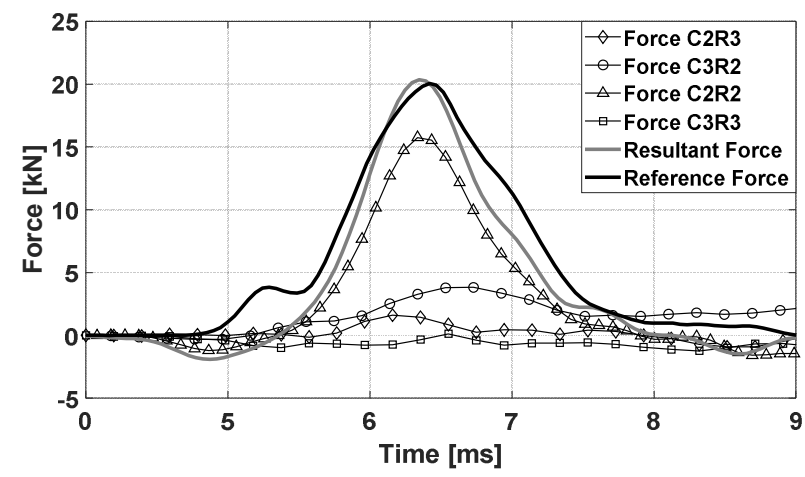

Fig. 10 Result of the impact test on a subset of four pixels. Force components recorded with the pixels belonging to columns $\mathrm{C} 2, \mathrm{C} 3$, and rows R2, R3. Resultant force (grey) and reference force (black).

A good agreement between the reference and resultant force is observed although the smaller values of impact forces are partially corrupted by crosstalk.

\section{Matrix B Pixel Performance Evaluation}

The matrix B backplane described in Section III has not been manufactured yet. However, test structures containing its pixel frontend electronics have been fabricated and characterized. The behavior of this frontend in both frequency and time domain is discussed in this subsection. The device under test consists of a matrix B pixel (Fig. 3(b)), loaded at the output by a discrete capacitor of about $30 \mathrm{pF}$, which is introduced to emulate $\mathrm{C}_{\mathrm{CLM}}$. Next, the output of the pixel is connected to a $\mathrm{Si}$ voltage buffer amplifier. Additional $5 \mathrm{pF}$ load are due to the input capacitance of the buffer, thus the total capacitive load is $35 \mathrm{pF}$ as discussed in Section III. The frequency response and the output noise are measured by means of the HP35670A Dynamic Signal Analyzer, connected to the amplifier. During these characterizations, the transistor $\mathrm{M}_{3}$ (Fig. 3(b)) is permanently turned on.

The measured frequency response is compared with circuit simulations in Fig. 11.

From the frequency response, an in band gain of $0.79 \mathrm{~V} / \mathrm{V}$ or $-2 \mathrm{~dB}$ can be extracted. The loading network constituted by $\mathrm{C}_{\mathrm{CLM}}$ and $\mathrm{M}_{3}$ determines the cut-off frequency of the source follower $\left(\mathrm{M}_{1}-\mathrm{M}_{2}\right)$, which is about $35 \mathrm{kHz}$ (Fig. 11). The current biasing the source follower is measured to be $85 \mu \mathrm{A}$.

A good agreement has been obtained between the measurement and the simulation results, as shown in the picture. The measured input-referred noise Power Spectral Density (PSD) is reported in Fig. 12. The frequency range analyzed, is only limited by the bandwidth of the instrument. The noise distribution is clearly dominated by the flicker noise introduced by the OTFTs.

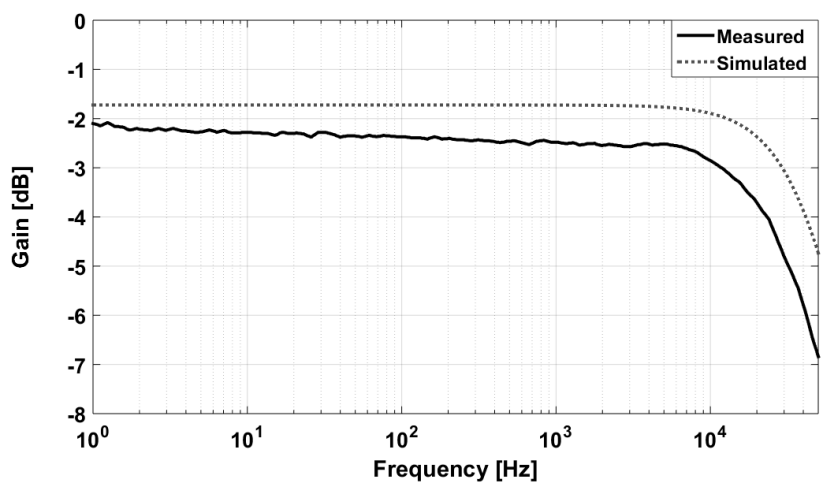

Fig. 11 Comparison between the measured (solid black) and simulated (dashed grey) frequency response of the matrix B frontend.

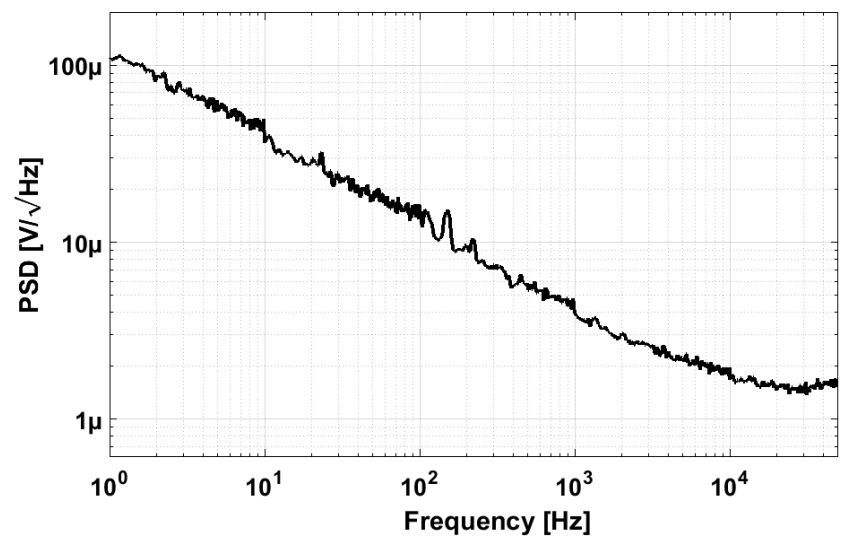

Fig. 12 Measured input-referred noise Power Spectral Density of the matrix B frontend.

The PSD reveals a noise level of $110 \mu \mathrm{V} / \mathrm{Hz}^{1 / 2}$ at $1 \mathrm{~Hz}$, which decreases down to $4.2 \mu \mathrm{V} / \mathrm{Hz}^{1 / 2}$ at $1 \mathrm{kHz}$. Moreover, the corner 
frequency falls approximately at $10 \mathrm{kHz}$. The integration of the PSD over the frequency, leads to an equivalent noise level at the input of the frontend equal to $451 \mu \mathrm{V}_{\text {rms. }}$. This result largely meets the noise requirement specified in Section III, and therefore the specification on the SNR is satisfied.

The performance of the matrix $\mathrm{B}$ frontend has also been evaluated in the time domain by means of its transient response. The input voltage of the source follower $\mathrm{M}_{1}-\mathrm{M}_{2}$ (Fig. 3(b)) has been brought to $5 \mathrm{~V} \mathrm{DC}$, which corresponds to the maximum expected input signal. The corresponding measured output voltage of the source follower in steady state is $4.10 \mathrm{~V}$ (grey dotted line in Fig. 13). During the transient measurement the switch $\mathrm{M}_{3}$ (Fig. 3(b)) is turned on for $20 \mu$ s ("row addressing time"), to enable the readout. The measured transient response of the pixel output is reported in Fig. 13 (continuous line). A settling time of $13.8 \mu \mathrm{s}$ is required to reach the final voltage value with $\delta=5 \%$ accuracy.

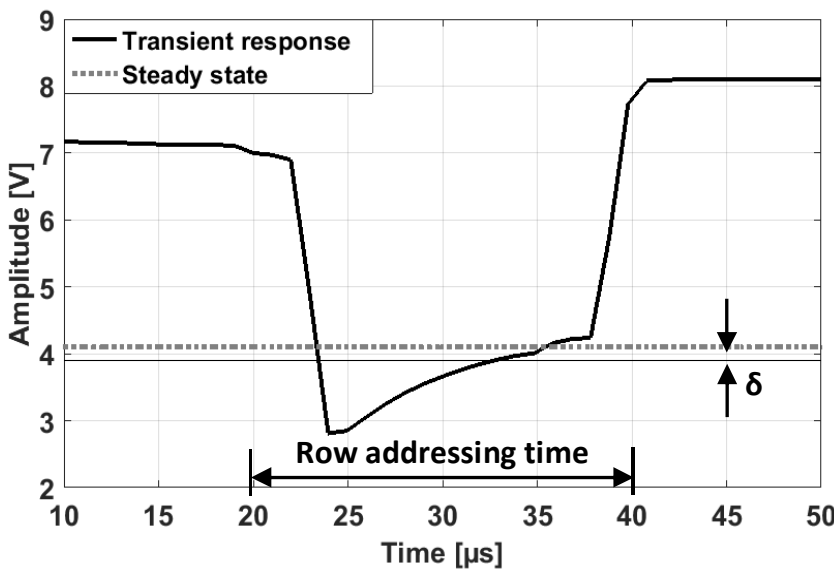

Fig. 13 Measured transient response (solid black) and output voltage at steady state condition (dashed grey). $\delta$ indicates the allowed error margin for settling.

\section{CONCLUSIONS}

In this work, we designed and simulated two different pressure-sensing systems that are built laminating an organic thin film transistor frontend matrix with a piezoelectric sensor matrix. The systems differ for the schematic of the pixel in the frontend matrix (matrix A and matrix B). Simulation results show that $78.6 \mathrm{~dB}$ of maximum SNR and a readout speed of $5 \mathrm{kframe} / \mathrm{s}$ can be achieved with matrix B. A complete electric characterization of both pixel designs has also been performed. Experimental results prove the capability of the matrix A to reconstruct correctly the profiles of impact forces up to $50 \mathrm{kN}$ applied by dropping a weight on the sensor. This simple and compact solution, however, suffers from cross-talk issues. The frontend electronics implemented in matrix B, solves these cross-talk problems. With a measured input referred equivalent noise of $451 \mu \mathrm{V}_{\text {rms }}$, and a $5 \%$ settling time of $13.8 \mu \mathrm{s}$, its pixel frontend fulfills the requirements dictated by the target applications.

\section{ACKNOWLEDGMENT}

The authors would like to acknowledge the financial support of the European Commission for the projects ATLASS (Horizon 2020, Nanotechnologies, Advanced Material and Production theme, contract $n^{\circ} 636130$ ).

\section{REFERENCES}

[1] T. R. Bennett, J. Wu, N. Kehtarnavaz, and R. Jafari, "Inertial measurement unit-based wearable computers for assisted living applications: A signal processing perspective,” IEEE Signal Processing Magazine, vol. 33, no. 2, pp. 28-35, March 2016.

[2] E. Cippitelli, F. Fioranelli, E. Gambi, and S. Spinsante, "Radar and rgbdepth sensors for fall detection: A review," IEEE Sensors Journal, vol. 17, no. 12, pp. 3585-3604, June 2017.

[3] E. E. Stone and M. Skubic, "Fall detection in homes of older adults using the microsoft kinect," IEEE journal of biomedical and health informatics, vol. 19, no. 1, pp. 290-301, January 2015.

[4] M. S. Khan, M. Yu, P. Feng, L. Wang, and J. Chambers, "An unsupervised acoustic fall detection system using source separation for sound interference suppression," Signal processing, vol. 110, pp. 199210, August 2014.

[5] G. Feng, J. Mai, Z. Ban, X. Guo, and G. Wang, "Floor pressure imaging for fall detection with fiber-optic sensors," IEEE Pervasive Computing, vol. 15, no. 2, pp. 40-47, April 2016.

[6] T. Someya, T. Sekitani, S. Iba, Y. Kato, H. Kawaguchi, and T. Sakurai, "A large-area, flexible pressure sensor matrix with organic field-effect transistors for artificial skin applications," Proc. Nat. Acad. Sci. USA, vol. 101, pp. 9966-9970, July 2004.

[7] M. Kaltenbrunner, T. Sekitani, J. Reeder, T. Yokota, K. Kuribara, T. Tokuhara, M. Drack, R. Schwodiauer, I. Graz, S. B. Gogonea, S. Bauer, T. Someya, "An ultra-lightweight design for imperceptible plastic electronics”, Nature 499, pp. 458-463, 2013.

[8] G. Schwartz, B. C.K. Tee, J. Mei, A. L. Appleton, D. H. Kim, H. Wang, Z. Bao, "Flexible polymer transistors with high pressure sensitivity for application in electronic skin and health monitoring", Nature Communications 4, 2013.

[9] T. Yokota, T. Sekitani, Naoya Take, U. Zschieschang, H. Klauk, K. Takimiya, T.C. Huang, M. Takamiya, T. Sakuai, T. Someya, "Sheet-Type Flexible Organic Active Matrix Amplifier System Using Pseudo-CMOS Circuits with Floating-Gate Structure. IEEE Trans. Electron Devices, vol. 59, no.12, pp. 3434-3440, December 2012.

[10] M. Zirkl, A. Sawatdee, U. Helbig, M. Krause et al, "An All-Printed Ferroelectric Active Matric Sensor Network Based on Only Five Functional Materials Forming a Touchless Control Interface," Advanced Materials, vol. 23, no. 18, pp. 2069-2074, May 2011.

[11] I.Chartier, S.Jacob, M. Charbonneau, A. Aliane, A. Plihon, R. Coppard, R.Gwoziecki, J.M. Verilhac, C. Serbutoviez, O. Dhez, E. Cantatore, F.D. Dos Santos, "Printed OTFT complementary circuits and matrix for Smart Sensing Surface applications", $44^{\text {th }}$ European Solide State Device Research Conference (ESSDERC), September 2014.

[12] M.Fattori, E. Cantatore, G. Pauer, T. Agostinelli, B. Stadlober, H. Gold, "Flexible pressure and proximity sensor surfaces manufactured with organic materials", $7^{\text {th }}$ IEEE International Workshop on Advances in Sensors and Interfaces (IWASI), June 2017.

[13] S. Ravinder, M. Valle, "Robotic Tactile Sensing - Technologies and Systems", Springer, 2013.

[14] T. Agostinelli, B. Yaglioglu,I. Horne, S. Markham, "Low Leakage Organic Backplanes for Low Power and High Pixel Density Flexible Displays". SID Symposium Digest of Technical Papers, vol. 47, no. 1, pp. 1523-1525, May 2016.

[15] D. MacDougall, W. B. Crummett and et al. , "Guidelines for data acquisition and data quality evaluation in envionmental chemistry". Analytical Chemistry, vol. 52, no. 14, pp. 2242-2249, December 1980. 


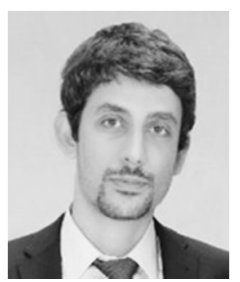

Marco Fattori was born in Rieti, Italy, in 1990. He received the M.S degree in electronic engineering in the 2014 from the Università degli Studi dell'Aquila, Italy. He is currently working towards the Ph.D. in the Mixed-Signal and Microelectronics group, department of Electrical Engineering, Eindhoven University of Technology (TUE), Eindhoven, The Netherlands. His research interest includes the analog and mixed signal circuit design on organic electronics, for flexible and large-area applications.

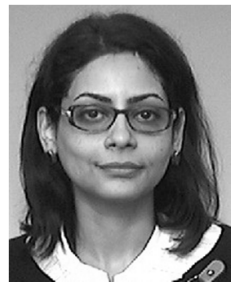

Sahel Abdinia received her $\mathrm{PhD}$ degree in electrical engineering from Eindhoven University of Technology (TUE) in 2014, where she carried research on circuit design using complementary organic transistors. Subsequently, she worked on biomedical applications as a circuit designer in Philips Research and Medtronic Eindhoven Medtronic Design Center (MEDC). Since December 2016, she holds an assistant professorship position in Mixed-signal Microelectronics group at TUE. Her research is focused on mixed-signal circuit design using emerging technologies such as organic and IGZO TFTs, and, most recently, on electronics for flexible and wearable biomedical applications.

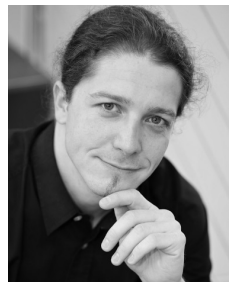

Gernot Pauer received the Ph.D. degree in 2005, at the University of Technology in Graz, at the Institute for Solid State Physics. Since 2005 he is employed at Concept Tech, a supplier for automotive engineering and testing services in Gratkorn/Graz, which is now part of the Altran Group. As Head of Industrial Engineering and Product Development, his working areas comprise FE-simulation methods in the context of vehicle- and pedestrian-safety, material characterization, and development of automotive test equipment and customized testing solutions.

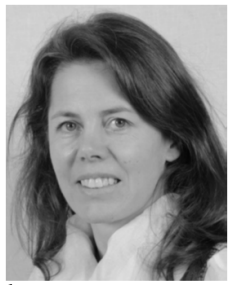

Barbara Stadlober received the Ph.D. degree in 1996. She is Head of the Research Group "Micro-\& Nanostructuring" at the Institute of Surface Technologies and Photonics of the JOANNEUM RESEARCH Forschungsgesellschaft mbH (JR), Graz/Weiz, Austria. She has a background in low temperature and solid state physics, was part of the reliability team at Infineon Technologies Austria in Villach and joined JR in 2003 for building up the group "Organic Field Effect Transistors". Her current interests range from organic and printed electronics over R2Rnanopatterning to large-area physical sensors and biomimetic structures.

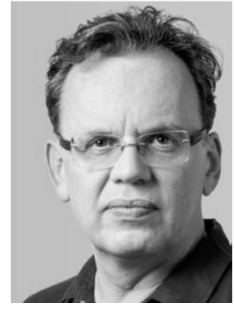

Herbert Gold received the Ph.D. degree investigating thin-film materials of silicon semiconductor technology, in 1999. He had been a development engineer at the CRTmaker LG.Philips-Displays in Austria and in the U.K. until 2004. Since then he has been working as a scientist at Joanneum Research in the field of organic electronics particularly in designing and manufacturing imprint-tools and in management of EU-funded projects PolyNet, POLARIC, and ATLASS

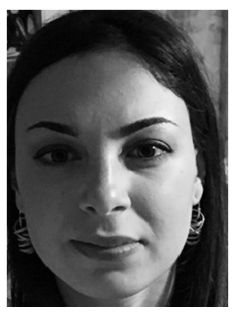

Josephine Socratous holds a BA and MSci in Experimental and Theoretical Physics from the University of Cambridge. Her PhD thesis was on amorphous metal oxide semiconductors for thin-film transistor applications at the Optoelectronics group, Cavendish Laboratory, University of Cambridge. Since 2015, she has been working as a research engineer at FlexEnable Ltd. She is currently working on improving the performance and reliability of organic thin film transistors as well as managing EU-funded projects.

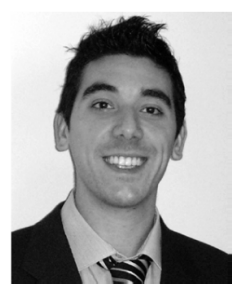

Fabrizio Torricelli received the Ph.D. degree from the University of Brescia, Brescia, Italy, in 2010. He was a PostDoctoral Fellow with the Eindhoven University of Technology, Eindhoven, The Netherlands, from 2010 to 2012. He is currently an Assistant Professor with the University of Brescia. His current research interests include the theoretical and experimental analysis of organic and amorphousoxide materials, the design of optoelectronic devices, and nonvolatile memories.

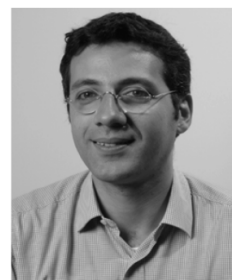

Eugenio Cantatore (F'16) received his Master's and Ph.D. Degree in Electrical Engineering from Politecnico di Bari, in 1993 and 1997 respectively. From 1997 to 1999 he was fellow at the European Laboratory for Particle Physics (CERN), Geneva. In 1999 he moved to Philips Research, Eindhoven, as senior scientist and in 2007 joined the Eindhoven University of Technology, where he is full professor since 2016. His research interests include the design and characterization of electronic circuits exploiting emerging technologies and the design of ultra-low power micro-systems. He authored or co-authored more than 140 papers in journals and conference proceedings, and 13 patents or patent applications. He is active in the Technical Program Committees of IWASI, ESSDERC, ESSCIRC and ISSCC. From 2013 till 2016 he has been chair of the Technology Directions subcommittee, and he is presently Program vice-chair of ISSCC. In 2006 he received from ISSCC the Beatrice Winner Award for Editorial Excellence and was nominated in the Scientific American top 50 list. He received the Philips Research Invention Award in 2007, the Best Paper Award from ESSDERC 2012 and the Distinguished Technical Paper Award from ISSCC 2015. 